\title{
Association Between Retroperitoneal Lymphadenectomy and Survival Benefit in the Endometrioid-Type Endometrial Carcinoma Patients
}

\author{
Fatemeh Cheraghi ${ }^{1, *}$; Mitra Modaress Gilani ${ }^{2}$; Fatemeh Ghaemmaghami ${ }^{2}$; Azamsadat \\ Mousavi $^{2}$

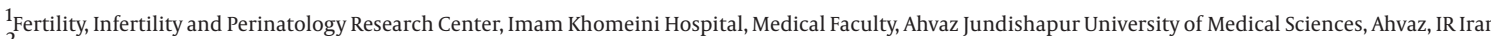 \\ ${ }^{2}$ Vali-e-Asr Hospital, Medical Faculty, Tehran University of Medical Sciences, Tehran, IR Iran \\ ${ }^{*}$ Corresponding author: Fatemeh Cheraghi, Fertility, Infertility and Perinatology Research Center, Imam Khomeini Hospital, Medical Faculty, Ahvaz Jundishapur University of \\ Medical Sciences, Ahvaz, IR Iran. Tel: +98-9163064850, Fax: +98-2166909309, E-mail: cheraghfati@yahoo.com \\ Received: June 18, 2014; Accepted: December 26, 2014
}

\begin{abstract}
Background: Uterine cancer stays the most prevalent gynecologic malignancy in developed countries. Providing suitable solutions to treat this disease is required to the early diagnosis of this cancer.

Objectives: The aim of this study was to evaluate the effectiveness of multiple-site lymph node sampling in patients with the endometrioid type of endometrial cancer.

Patients and Methods: Demographic and clinicopathologic data were obtained from the Tehran Gynecology Oncology ward in Vali-e-Asr Hospital between 1999 and 2010. All the patients were divided into two groups. Group A $(n=102)$ : patients who underwent multiple-site lymph node sampling; and Group B $(\mathrm{n}=102)$ : patients who did not undergo multiple-site lymph node sampling. Survival curves were calculated using the Kaplan-Meier method, and the differences in survival rates were analyzed using the log-rank test.

Results: The median age was 55 years (range $=18-84$ ). The 5 -year progression-free survival rates of Groups A and B were $85 \%$ and $87 \%$, respectively $(\mathrm{P}=0.456)$. In addition, the 5 -year overall survival rates were $95 \%$ and $93.9 \%$, respectively $(\mathrm{P}=0.651)$. Subsequently, there was no significant difference in recurrence rates in retroperitoneal lymph nodes regardless of the completion of lymphadenectomy.

Conclusions: The obtained data showed no evidence of benefit in terms of overall or recurrence-free survival for pelvic lymphadenectomy in women with early endometrial cancer.
\end{abstract}

Keywords: Endometrial Neoplasms; Endometrial Type; Lymph Node Excision; Survival

\section{Background}

Uterine cancer remains the most prevalent gynecologic malignancy in developed countries. For example, the USA was estimated to have 40100 new cases of uterine cancer in 2008 (1). In the USA, the annual number of deaths resulting from this cancer has increased, with about 3000 in the 1980s, 5000 in the 1990s, and 7000 in more recent years (2). Regarding this increase, improvements are required in the early diagnosis and treatment of this cancer. Prior studies suggest that age, stage, histology, tumor grade, myometrial invasion, and nodal involvement are significant prognostic factors $(3,4)$. One of the problems in the optimum surgical treatment of endometrial cancer results from inconsistencies in staging and, in particular, lymph node evaluation. Several studies such as those conducted by the US National Comprehensive Cancer Network (5) and the American College of Obstetricians and Gynecologists (6) have suggested a therapeutic benefit associated with lymphadenectomy, whereas some other studies such as A Study in the Treatment of Endometrial Cancer (ASTEC) (7) have shown no survival benefit. In the present study, a large number of patients with the endometrioid type of endometrial cancer were analyzed retrospectively to evaluate the benefit of multiple-site lymph node sampling.

\section{Objectives}

The aim of this study was to evaluate the effectiveness of multiple-site lymph node sampling in patients with the endometrioid type of endometrial cancer.

\section{Patients and Methods}

Patients with preoperative endometrioid-type endometrial carcinoma thought to be clinically confined to the uterus (International Federation of Gynecology and Obstetrics [FIGO] stage I) and had been treated at Tehran Gynecology Oncology ward in Vali-e-Asr Hospital between 1999 and 2010 were assigned in two groups: Group A ( $\mathrm{n}=$ 102) underwent multiple-site lymph node sampling and Group B $(\mathrm{n}=102)$ did not. All the patients allocated to the multiple-site lymph node sampling arm received lymph-

Copyright (C) 2015, Ahvaz Jundishapur University of Medical Sciences. This is an open-access article distributed under the terms of the Creative Commons Attribution-NonCommercial 4.0 International License (http://creativecommons.org/licenses/by-nc/4.0/) which permits copy and redistribute the material just in noncommercial usages, provided the original work is properly cited. 
adenectomy, with a median of 24 pelvic lymph nodes removed (range $=16-30$ ).

Data were obtained from special oncology files, and the following features were recorded for each patient: age of diagnosis; parity; surgical procedure; substage; histology; grade; depth of myometrial invasion; size of the tumor; lymph vascular space invasion; lymph node status; adjuvant therapy; surgical morbidity; time to recurrence; date of death; and last follow-up. The patients were followed up every 3 months for the first 2 years, and every 6 months thereafter. The median follow-up period was 30 months (range $=3-108$ ).

Progression-free survival (PFS) was defined as the interval from the date of primary surgery until the recurrence or death or the last follow-up. The overall survival (OS) duration was determined as the time from the date of primary surgery until death or the last follow-up. PFS and OS curves were calculated using the Kaplan-Meier method, and significance was determined using the logrank test. Two-tailed tests at P values $<0.05$ were considered significant.

\section{Results}

The clinical and tumor characteristics of the eligible patients are listed in Table 1 . The mean follow-up was 60 months (36 - 108) for all the patients. The patients were well matched between the two arms in terms of clinicopathological features. The median age at the time of randomization was 54 years (range $=28-74$ ) for the group with multiple-site lymph node sampling and 53 years (range $=26-80$ ) for the group who did not undergo lymphadenectomy.

The histological cell type was the endometrioid type in the two groups. The depth of invasion was as follows for the group with multiple-site lymph node sampling (Group A): endometrial only: 11 (10/8\%); inner half of the myometrium: 60 (58.8\%); and outer half of the myometrium: 31 (30.4\%). The depth of invasion was as follows for the group without lymphadenectomy (Group B): endometrial only: 10 (9/8\%); inner half of the myometrium: 62 (60.8\%); and outer half of the myometrium: 30 (29.4\%).

The tumor grades were as follows for Group A versus Group B: 37 women (36.3\%) versus 35 (34.4\%) had tumor grade $1 ; 50$ (49\%) versus 54 (52.9\%) grade 2 ; and $15(14.7 \%)$ versus $13(12.7 \%)$ grade 3.

In Group A, 72 (70.6\%) patients were in stage I according to the FIGO, 10 (9.8\%) in stage II, and 20 (18.6\%) in stage III. In Group B, 70 (68.6\%) patients were in stage I according to the FIGO, 14 (13.7\%) in stage II, and 18 (17.7\%) in stage III.

The rates of adjuvant therapy (pelvic external beam, brachytherapy, chemotherapy, or a combination of chemotherapy and radiotherapy) were not different significantly between the two groups. The majority of the patients (69.3\%) in the multiple-site lymph node sampling group and $64 \%$ in the group without lymphadenectomy did not receive adjuvant therapy.

There was no statistically significant difference in the risk of death (OS) between the two groups after adjustment for important prognostic factors, including age and tumor grade ( $\mathrm{HR}=1.022,95 \% \mathrm{CI}$ : 0.51 to 2.44 ).

The 5-year OS rate of Group A was 95\%, compared with 93.9\% in Group B. On Kaplan-Meier analysis, the difference in OS between these groups was non-significant ( $P$ $=0.651$ ). Moreover, the 5-year PFS rate of Group A was 85\%, compared with $87 \%$ in Group B. The difference in PFS between these groups was also non-significant $(P=0.456)$ (Figure 1).

\begin{tabular}{lcc}
\hline \multicolumn{1}{l}{ Table 1. Demographic characteristics of the maternal and the Endometrial Neoplasms } & \\
\hline Characteristics & Lymphadenectomy Arm $^{\text {a }}$ & No Lymphadenectomy Arm $^{\text {a }}$ \\
\hline Median age & $54(28-74)$ & $53(26-60)$ \\
\hline Tumor grade & $37(36.3)$ & $35(34.4)$ \\
\hline $\begin{array}{l}\text { (well differentiated) } \\
2 \text { (moderately differentiated) }\end{array}$ & $50(49)$ & $54(52.9)$ \\
\hline 3 (poorly differentiated) & $15(14.7)$ & $13(12.7)$ \\
\hline Depth of invasion & $11(10.8)$ & $10(9.8)$ \\
\hline Endometrial only & $60(58.8)$ & $62(60.8)$ \\
\hline Inner half of the myometrium & $31(30.4)$ & $30(29.4)$ \\
\hline Outer half of the myometrium & & $70(68.6)$ \\
\hline FIGO ${ }^{b}$ stage & $72(70.6)$ & $14(13.7)$ \\
\hline I & $10(9.8)$ & $18(17.7)$ \\
\hline II & $20(18.6)$ & \\
\hline III & & \\
\hline
\end{tabular}

\footnotetext{
${ }^{\mathrm{a}} \mathrm{n}=102$.

b FIGO: International Federation of Gynecology and Obstetrics.
} 


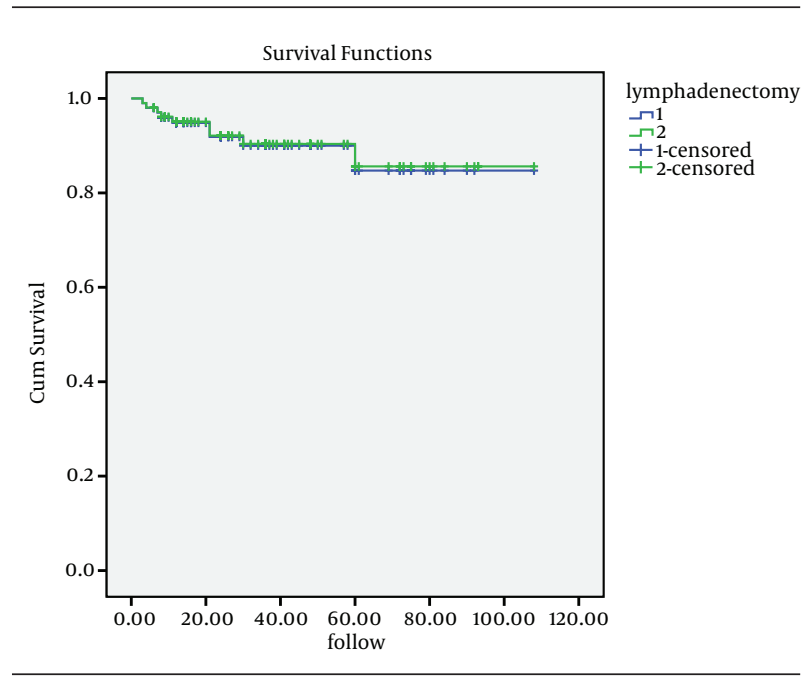

Figure 1. Disease-Free Survival for Patients with Clinical Early-Stage Endometrial Cancer Undergoing Multiple-Site Lymph Node Sampling versus Those with No Lymphadenectomy

Also, there was no statistically significant difference in the risk of disease recurrence between the two groups after adjustment for important prognostic factors, including age and tumor grade ( $\mathrm{HR}=1.024,95 \% \mathrm{CI}$ : 0.64 to 1.62$)$. There were $8(3 / 9 \%)$ postoperative complications in the multiple-lymph node dissection group: 4 cases of wound infection and 4 ileus episodes requiring prolonged hospital stay. No surgical mortality was seen, and no patient developed a major complication directly related to lymphadenectomy.

\section{Discussion}

Our findings revealed no association between survival and lymphadenectomy in patients with the endometrioid-type endometrial cancer. Comprehensive surgical staging for patients with endometrial cancer remains controversial and ranges from universal lymphadenectomy (8) to lymphatic assessment in only those with adverse risk factors (9).

Several investigators have recommended a therapeutic advantage associated with lymphadenectomy (10-14), while others have shown no survival advantage $(15,16)$. Obviously, there is still considerable controversy over the advantages and risks of lymph-node dissection in lowrisk (e.g. stage IA, all grades and stage IB, grades 1 and 2) patients $(17,18)$. The US National Cancer Institute database compared 12333 (31.3\%) women who underwent surgical staging procedures, including lymphadenectomy, with 27063 patients who did not receive lymphadenectomy and reported that the 5-year disease-specific survival of the women with disease stages I, II, III, and IV who underwent lymphadenectomy was $95.5 \%, 90.4 \%, 73.8 \%$, and $53.3 \%$, respectively, compared with $96.6 \%, 82.2 \%, 63.1 \%$, and $26.9 \%$ for those who did not have lymphadenectomy ( $\mathrm{P}>$ 0.05 for stage I and $\mathrm{P}<0.001$ for stages II-IV) (17). Additionally, the patients with stage I disease who did not undergo lymphadenectomy had a higher proportion of tumors with grade 1 histology or disease limited to the endometrium (or both) than those who had lymphadenectomy. In a subset of patients with stage I, grade 3 disease, those who underwent lymphadenectomy had better disease-specific survival than those who did not have lymphadenectomy (90\% vs. 85\%; P = 0.0001); however, no benefit for lymphadenectomy was identified for the patients with stage I, grade $1(P=0.26)$ and grade $2(P=0.14)$ disease $(17)$.

The initial consequences of ASTEC have been reported, showing that pelvic lymphadenectomy does not confer survival advantage (7). Although in this clinical trial over 700 patients underwent lymphadenectomy, it was difficult to highlight the value of lymphadenectomy in patients with low-risk endometrial uterine cancer (7).

Nonetheless, most academic centers in the USA advise lymphadenectomy as part of the staging procedure in all patients with endometrial uterine cancer (5). Moreover, discussion concerning the role of complete versus selective lymphadenectomy is still ongoing among those who recommend the use of lymphadenectomy for patients with uterine cancer. For example, the Gynecologic Oncology Group (GOG) completed a trial on intermediate-risk endometrial uterine cancer, which required the study participants to undergo selective, bilateral pelvic and para-aortic lymphadenectomy (19). By contrast, the US National Comprehensive Cancer Network advised lymph node dissection rather than nodal sampling in patients undergoing primary surgical management of endometrioid uterine cancer (5). However, our study showed no evidence of benefits in terms of overall or recurrence-free survival for pelvic lymphadenectomy in women with early endometrial cancer.

The weaknesses of this study include the retrospective nature and lack of a central pathology review. The retrospective nature does introduce potential selection bias; however, it should statistically affect all patients equally. Although there was not a 100\% central pathologic review, all the surgeries were performed at one institution in which a pathologist specializing in gynecologic pathology reviewed all pathology at the time of diagnosis and at a gynecologic/ oncology conference.

\section{Acknowledgements}

The authors wish to acknowledge the efforts of Dr. Masoud Hemadi (Vice Chief of the Fertility, Infertility and Perinatology Research Center, Ahvaz Jundishapur University of Medical Sciences [AJUMS], Ahvaz, IR Iran) for his generous help in processing the study.

\section{Authors' Contributions}

All the authors contributed equally to the manuscript.

\section{References}

1. Jemal A, Siegel R, Ward E, Hao Y, Xu J, Murray T, et al. Cancer statistics, 2008. CA Cancer J Clin. 2008;58(2):71-96. 
2. Jemal A, Siegel R, Ward E, Murray T, Xu J, Smigal C, et al. Cancer statistics, 2006. CA Cancer J Clin. 2006;56(2):106-30.

3. Creasman WT, Morrow CP, Bundy BN, Homesley HD, Graham JE Heller PB. Surgical pathologic spread patterns of endometrial cancer. A Gynecologic Oncology Group Study. Cancer. 1987;60(8 Suppl):2035-41.

4. Creasman WT. Announcement. FIGO stages-1988 Revisions. Gynecol Oncol. 1989;35:125-7.

5. Greer BE, Koh WJ, Abu-Rustum N, Bookman MA, Bristow $\mathrm{RE}$, Campos S, et al. Uterine cancers. J Natl Compr Canc Netw. 2006;4(5):438-62.

6. American College of O, Gynecologists.. ACOG practice bulletin, clinical management guidelines for obstetrician-gynecologists, number 65, August 2005: management of endometrial cancer. Obstet Gynecol. 2005;106(2):413-25.

7. Kitchener H, Redman CW, Swart AM, Amos CL. on behalf of ASTEC Study Group. ASTEC. A study in the treatment of endometrial cancer. A randomised trial of lymphadenectomy in the treatment of endometrial cancer. Gynecol Oncol. 2006;101(Suppl 1):S21-S22.

8. Huh WK, Straughn JJ, Kelly FJ, Kilgore LC. Endometrial carcinoma. Curr Treat Options Oncol. 2001;2(2):129-35.

9. Aalders JG, Thomas G. Endometrial cancer--revisiting the importance of pelvic and para aortic lymph nodes. Gynecol Oncol. 2007;104(1):222-31.

10. Kilgore LC, Partridge EE, Alvarez RD, Austin JM, Shingleton HM, Noojin F3, et al. Adenocarcinoma of the endometrium: survival comparisons of patients with and without pelvic node sampling. Gynecol Oncol. 1995;56(1):29-33.

11. Mohan DS, Samuels MA, Selim MA, Shalodi AD, Ellis RJ, Samuels $\mathrm{JR}$, et al. Long-term outcomes of therapeutic pelvic lymphadenectomy for stage I endometrial adenocarcinoma. Gynecol Oncol. 1998;70(2):165-71.
12. Cragun JM, Havrilesky LJ, Calingaert B, Synan I, Secord AA, Soper JT, et al. Retrospective analysis of selective lymphadenectomy in apparent early-stage endometrial cancer. J Clin Oncol. 2005;23(16):3668-75.

13. Trimble EL, Kosary C, Park RC. Lymph node sampling and survival in endometrial cancer. Gynecol Oncol. 1998;71(3):340-3.

14. Mariani A, Webb MJ, Keeney GL, Haddock MG, Calori G, Podratz KC. Low-risk corpus cancer: is lymphadenectomy or radiotherapy necessary? Am J Obstet Gynecol. 2000;182(6):1506-19.

15. Carey MS, O'Connell GJ, Johanson CR, Goodyear MD, Murphy KJ Daya DM, et al. Good outcome associated with a standardized treatment protocol using selective postoperative radiation in patients with clinical stage I adenocarcinoma of the endometrium. Gynecol Oncol. 1995;57(2):138-44.

16. Eltabbakh GH, Piver MS, Hempling RE, Shin KH. Excellent longterm survival and absence of vaginal recurrences in 332 patients with low-risk stage I endometrial adenocarcinoma treated with hysterectomy and vaginal brachytherapy without formal staging lymph node sampling: report of a prospective trial. Int J Radiat Oncol Biol Phys. 1997;38(2):373-80.

17. Chan JK, Wu H, Cheung MK, Shin JY, Osann K, Kapp DS. The outcomes of 27,063 women with unstaged endometrioid uterine cancer. Gynecol Oncol. 2007;106(2):282-8.

18. Chan JK, Cheung MK, Huh WK, Osann K, Husain A, Teng NN, et al. Therapeutic role of lymph node resection in endometrioid corpus cancer: a study of 12,333 patients. Cancer. 2006; 107(8):1823-30.

19. Keys HM, Roberts JA, Brunetto VL, Zaino RJ, Spirtos NM, Bloss JD, et al. A phase III trial of surgery with or without adjunctive external pelvic radiation therapy in intermediate risk endometrial adenocarcinoma: a Gynecologic Oncology Group study. Gynecol Oncol. 2004;92(3):744-51. 